Rapid Communications

\title{
Nuclease Tolerant FRET Probe Based on DNA-Quantum Dot Conjugation
}

\author{
Daisuke Onoshima, ${ }^{* 1, * 2 \dagger}$ Noritada KAJI, ${ }^{* 1, * 2}$ Manabu ToKeSHI, $* 1, * 2$ and Yoshinobu BABA $* 1, * 2, * 3, * 4, * 5$ \\ *1 Department of Applied Chemistry, Graduate School of Engineering, Nagoya University, \\ Nagoya 464-8603, Japan \\ *2 MEXT Innovative Research Center for Preventive Medical Engineering, Nagoya University, \\ Nagoya 464-8603, Japan \\ *3 Plasma Nanotechnology Research Center, Nagoya University, Nagoya 464-8603, Japan \\ *4 Health Technology Research Center, National Institute of Advanced Industrial Science and Technology (AIST), \\ Takamatsu 761-0395, Japan \\ *5 Institute for Molecular Science, National Institutes of Natural Sciences, Okazaki 444-8585, Japan
}

\begin{abstract}
We have developed a fluorescence resonance energy transfer (FRET) probe based on the conjugation of a quantum dot (QD) with dye (YOYO-3) intercalated DNA. The FRET-inducing electrostatic coupling of DNA and the QD made structural changes to the QD-DNA conjugates, which significantly prevented an enzymatic reaction between the DNA and a conventional restriction endonuclease (EcoRI).
\end{abstract}

(Received December 26, 2007; Accepted January 11, 2008; Published February 10, 2008)

Hybrid materials consisting of quantum dots (QDs) coupled to peptides, DNA and proteins are currently of great interest in the developing research area of nanobiotechnology. ${ }^{1-7}$ QD-based FRET (fluorescence resonance energy transfer) probes have recently been reported, including QD-conjugated hybridization probes for the preliminary screening of siRNA sequences ${ }^{8}$ and an ultrasensitive DNA nanosensor with a single quantum dot, ${ }^{9}$ which are benefited by the use of the unique photophysical properties of QDs and their conjugates.

Actually, QDs are applied as an excellent donor in FRET due to their narrow emission and broad excitation spectra, enabling an effective separation of the donor and the acceptor fluorescence, and the selection of a wide range of excitation wavelengths to reduce the background. By placing both donor and acceptor fluorophores at precise locations on the molecule of interest, information on distance and angle changes between the two fluorophores, or conformational and structural changes of the labeled molecules can be extracted through FRET measurements. ${ }^{10-13}$

A more specific example of one type of measurement of current interest may be drawn from the current work of Ho et al. ${ }^{14}$ on the intracellular stability and unpacking of a DNA molecule. They used a QD-mediated FRET to determine the dynamic stability and the composition of plasmid DNA during intracellular transport, and achieved the precise detection of discrete changes in the nanocomplex state against various intracellular microenvironments. This approach provides a convenient method to follow the intra- or intercellular trafficking of DNA nanocomplexes over time. However, these nucleic acids are very susceptible to a nuclease attack under physiological conditions. ${ }^{15}$ To overcome this problem, we have recently developed a unique FRET-inducing QD-DNA conjugate that is tolerant to nuclease digestion. Here, we report on a FRET analysis for structural changes of the QD-DNA

$\doteqdot$ To whom correspondence should be addressed. E-mail: onoshima.daisuke@d.mbox.nagoya-u.ac.jp conjugates and the inhibitory effect on the enzymatic reaction by dye binding.

QD-DNA conjugates were prepared by varying the QD/DNA ratio $(\mathrm{QD} / \mathrm{DNA}=17,168$ and 1678$)$ and using gel electrophoresis to separate the products. The method used for the conjugation of QD and DNA is schematically depicted in Fig. 1.16 In brief, 3'-biotinylated 14-mer oligonucleotide (5'GGGCGGCGACCTAA3'-biotin, Sigma Genosys) was hybridized with complementary $5^{\prime}$-overhang (12 base) of $\lambda$ DNA (Takara Bio) and linked by ligation. The biotinylated $\lambda$ DNA $(0.30,3.0$ and $30 \mathrm{pM})$ was reacted with streptavidin-coated CdSe/ZnS QD (0.5 nM, Qdot 605 Streptavidin Conjugate, Quantum Dot Corp.) in $82 \mathrm{mM} \mathrm{NaHCO}$ (pH 8.0). Each QD typically has 5-10 streptavidins on its surface (User Manual PN 90-0003J Rev 9.1), thus providing several types of

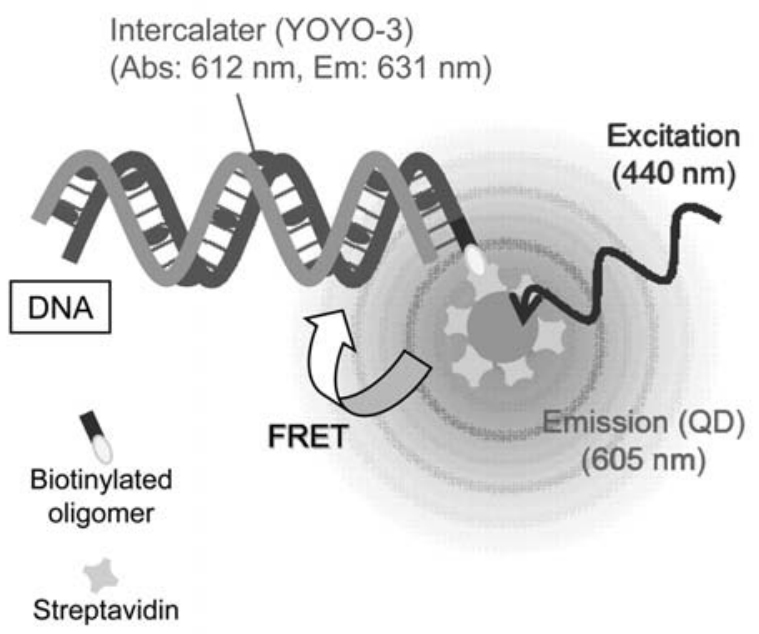

Fig. 1 Schematic of the FRET between the QD donor and the DNA-intercalating dye (YOYO-3) acceptor. 


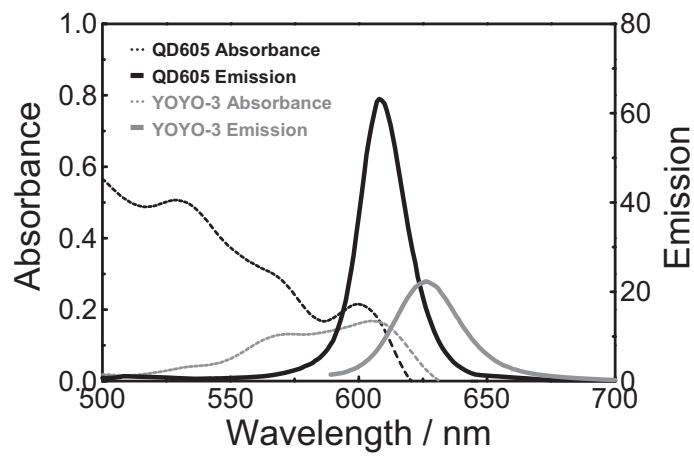

Fig. 2 Absorption (left scale) and emission (right scale) spectra of streptavidin-coated CdSe/ZnS Qdot 605 and YOYO-3 labelled $\lambda$ DNA.

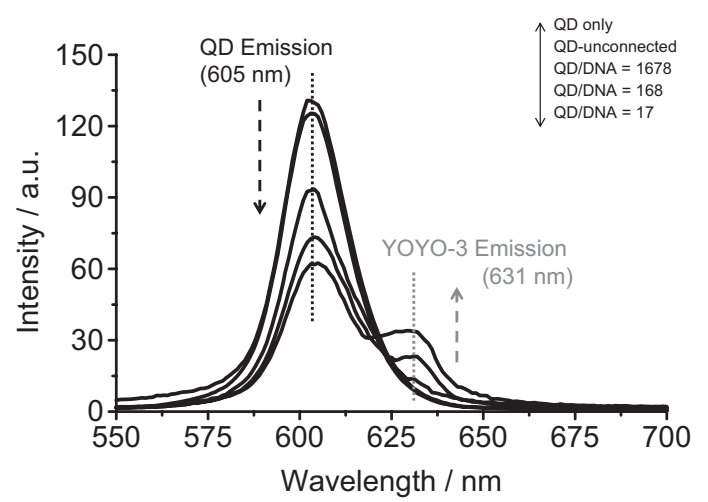

Fig. 3 Fluorescence spectra (excited at $440 \mathrm{~nm}$ ) of the QD-DNA conjugates at different QD/DNA ratios.

QD-DNA conjugates, which have different numbers of DNA molecules bound on the surface of a single QD. For a spectral fluorescence analysis of the conjugates, YOYO-3 iodide (YOYO-3, the dimmer of the asymmetrical cyanine dye, Molecular Probes) was intercalated to the DNA molecules with a ratio of 1:30 (dye molecules to DNA base pairs (bp)). Figure 2 shows the absorbance and emission features of the QD and YOYO-3 dye. The QD emission overlaps the absorption spectrum of the dye, suggesting that an efficient FRET between the QD donor and the YOYO-3 dye acceptor can take place. All measurements were carried out at room temperature.

Fluorescence spectra of the QD-DNA conjugates at different ratios of DNA to QD are shown in Fig. 3. They were recorded under excitation at $440 \mathrm{~nm}$ on a JASCO FP-6500 spectrofluorometer. Although the fluorescence intensity of QD $(605 \mathrm{~nm})$ progressively decreased with increased concentration of dye-labelled DNA, a new fluorescence band (631 nm, characteristic to YOYO-3) appeared, and increased in intensity. As a control, an unconnected mixture of the QD and the DNA (not biotinylated) was also tested (Fig. 3, QD-unconnected). No fluorescence intensity originating from the direct excitation of the YOYO-3 dye was observed, consistent with the fact that the dye does not absorb at the excitation wavelength of the QD. The estimated FRET efficiency, defined as $I_{\mathrm{A}} /\left(I_{\mathrm{A}}+I_{\mathrm{D}}\right)$, where $I_{\mathrm{A}}$ and $I_{\mathrm{D}}$ are the acceptor and donor intensities, increased with the ratio of DNA to QD (Fig. 4). This may be explained because the more DNA molecules were bound to QD, resulting in an increase in the number of dyes near the surface of the QD. The average surface coverage of the DNA was estimated to be only 1 to 5 molecules per particle (measured by extending and

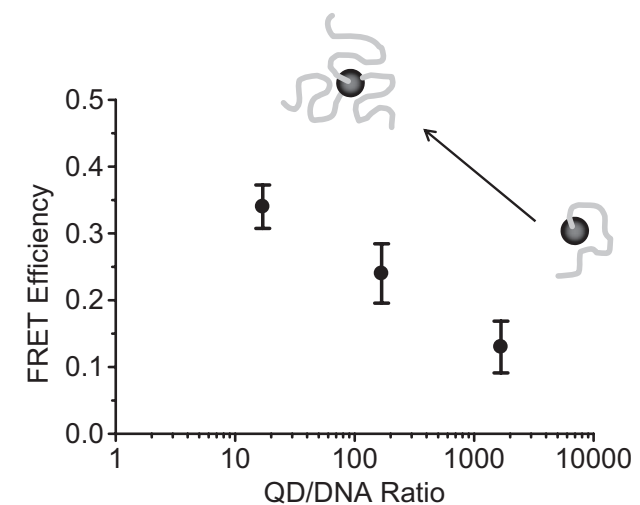

Fig. 4 Plot of the FRET efficiency versus the QD/DNA ratio. The inset is an explanatory drawing corresponding to each group of ratios. The error bars indicate the standard deviation.

observing the DNA molecules of the conjugates directly, see Ref. 16). It is most likely that QD-DNA conjugates may aggregate due to an interaction between the QD and the DNA backbone at a low DNA/QD ratio $(\leq 1) .{ }^{13}$ This is a common feature of QD-biomolecule conjugates that have been used in FRET studies. ${ }^{1,2} \quad$ Adsorptive interactions between oligonucleotides and QDs have also been reported and rationalized in terms of a hydrogen-bonding model. Algar and Krull $^{10}$ showed that conjugated oligonucleotides adopt a conformation that lies along the surface of the QD. The formation of these coupled structures is supposed to reduce the donor-acceptor distance and to improve the FRET efficiency.

We have also studied the induced-coupling of the dye-labelled DNA molecules to the QD, and evaluated the effect on DNA digestion by a conventional electrophoresis analysis. A typical gel image ( $0.8 \%$ agarose) is shown in Fig. 5 for a comparison of the mobility of related samples. The binding of YOYO-3 dye decreased the charge of the resulting QD-DNA conjugates and their electrophoretic mobility decreased. It is well known that DNA molecules stained with intercalating dyes show slower migration, because positively charged cyanine dyes can cancel out the negative charge of the DNA backbone. ${ }^{17}$ More importantly, the combination of QD-DNA conjugates with YOYO-3 dye caused a marked reaction inhibition with a restriction endonuclease (EcoRI, recognition site: GAATTC). Despite the fact that QD-DNA conjugates (without dye, lane 2) and $\lambda$ DNA (with dye, lane 3) show DNA digestion, the respective digested $\lambda$ DNA fragments of 21226, 7421, 5804, 5643, 4878 and $3530 \mathrm{bp}$, the QD-DNA conjugates (with dye, lane 4), show no sign of the digestion.

In recent years, an inhibitory effect on DNA digestion, which is attributed to an alteration in the DNA conformation due to binding of the intercalating dye, has been reported. ${ }^{18}$ Matsuura et al. ${ }^{19}$ presented the results that when the dye concentration was higher than a dye:nucleotide pair ratio of 1:20, a nuclease did not digest DNA molecules in the same way as unstained DNA. In our present research, where the ratio of 1:30 does not apply to this case, however, the inhibition surely occurred. These results indicate that there could be an additional electrostatic coupling between the YOYO-3 dye and the QD. This coupling may induce significant changes in the conformation or the structure of the QD-DNA conjugates, which would prevent an enzymatic reaction with nuclease molecules. Actually, the electrophoretic pattern seen in lane 5 is quite different from that of the original QD-DNA conjugates without a dye (lane 6). 


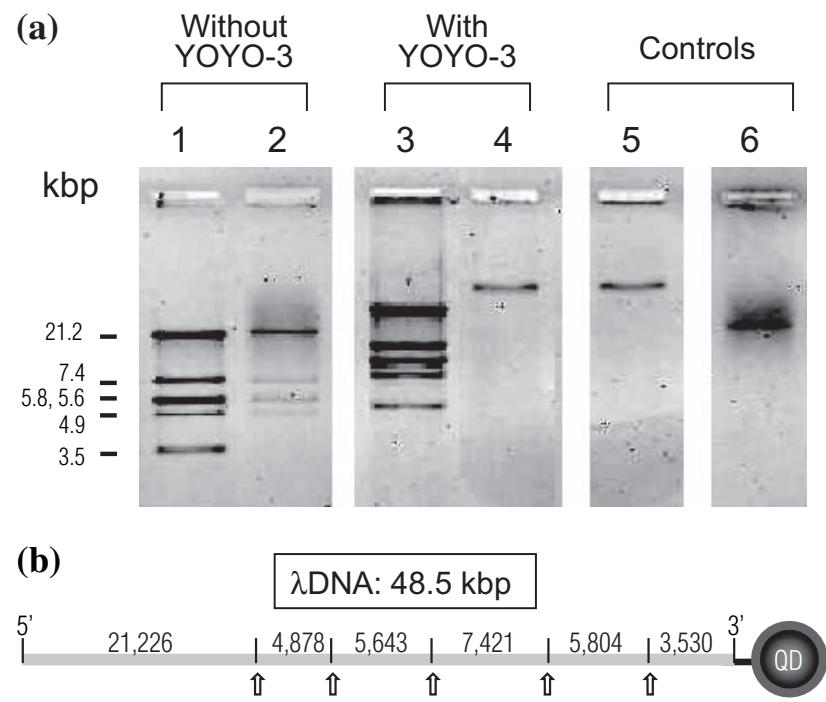

Fig. 5 Effect of YOYO-3 dye on DNA digestion by EcoRI. (a) The digested molecules were analyzed using agarose gel electrophoresis: 1, 3, $3.0 \mathrm{pM}$ $\lambda$ DNA; 2, 4-6, QD-DNA conjugate $(\mathrm{QD} / \mathrm{DNA}=168,0.3 \mathrm{pM} \lambda \mathrm{DNA})$. Lanes 5 and 6 are the controls in the absence of EcoRI (5, stained; 6, original). (b) Restriction-sites (GAATTC) in the QD-DNA conjugate.

On the other hand, the connection between the QD and DNA molecule must have been critical for this nuclease-tolerance property, because the unconnected mixture of QD and stained DNA (not biotinylated) shows decent DNA digestion (data not shown). Given the popularity of QD-mediated FRET measurements and the number of applications employing QD bioconjugates, the QD-DNA conjugate and an inducedtolerance property by dye binding are thought to be noticed from the viewpoint of utilization of the QD-conjugated functional materials in the cells or living organisms.

\section{Acknowledgements}

This study was performed through Special Coordination Funds for Promoting Science and Technology of the Ministry of Education, Culture, Sports, Science and Technology, the Japanese Government.

\section{References}

1. I. L. Medintz, A. R. Clapp, H. Mattoussi, E. R. Goldman, B. Fisher, and J. M. Mauro, Nat. Mater., 2003, 2, 630.

2. N. N. Mamedova, N. A. Kotov, A. L. Rogach, and J. Studer, Nano. Lett., 2001, 1, 281.

3. N. Kaji, M. Tokeshi, and Y. Baba, Anal. Sci., 2007, 23, 21.

4. R. Bakalova, Z. Zhelev, H. Ohba, and Y. Baba, J. Am. Chem. Soc., 2005, 127, 9328.

5. I. L. Medintz, L. Berti, T. Pons, A. F. Grimes, D. S. English, A. Alessandrini, P. Facci, and H. Mattoussi, Nano Lett., 2007, 7, 1741.

6. H. Peng, L. Zhang, T. H. M. Kjällman, C. Soeller, and J. T. Sejdic, J. Am. Chem. Soc., 2006, 129, 3048.

7. A. Fu, C. M. Micheel, J. Cha, H. Chang, H. Yang, and A. P. Alivisatos, J. Am. Chem. Soc., 2004, 126, 10832.

8. R. Bakalova, Z. Zhelev, H. Ohba, and Y. Baba, J. Am. Chem. Soc., 2005, 127, 11328.

9. C. Y. Zhang, H. C. Yeh, M. T. Kuroki, and T. H. Wang, Nat. Mater., 2005, 4, 826.

10. W. R. Algar and U. J. Krull, Langmuir, 2006, 22, 11346.

11. F. Patolsky, R. Gill, Y. Weizmann, T. Mokari, U. Banin, and I. Willner, J. Am. Chem. Soc., 2003, 125, 13918.

12. R. Gill, I. Willner, I. Shweky, and U. Banin, J. Phys. Chem. $B, \mathbf{2 0 0 5}, 109,23715$.

13. D. Zhou, J. D. Piper, C. Abell, D. Klenerman, D. J. Kang, and L. Ying, Chem. Commun., 2005, 4807.

14. Y. P. Ho, H. H. Chen, K. W. Leong, and T. H. Wang, J. Control. Release, 2006, 116, 83.

15. A. Shoji, M. Kuwahara, H. Ozaki, and H. Sawai, J. Am. Chem. Soc., 2007, 129, 1456.

16. D. Onoshima, N. Kaji, M. Tokeshi, and Y. Baba, in "Micro Total Analysis System 2007”, ed. J. L. Viovy, P. Tabeling, S. Descroix, and L. Mlaquin, 2007, Vol. 2, 1619 - 1621.

17. C. Carlsson, M. Jonsson, and B. Akerman, Nucleic Acids Res., 1995, 23, 2413.

18. C. Parolin, A. Montecucco, G. Ciarrocchi, G. P. Noy, S. Valisena, M. Palumbo, and G. Palu, FEMS Microbiol. Lett., 1990, 68, 341 .

19. S. Matsuura, J. Komatsu, K. Hirano, H. Yasuda, K. Takashima, S. Katsra, and A. Mizuno, Nucleic Acids Res., 2001, 29, e79. 